\title{
Improving efficiency for an open-loop controlled locomotion with a pulsed actuation
}

\author{
Jonathan Hunt, Fabio Giardina, Andre Rosendo Member, IEEE, Fumiya Iida Member, IEEE
}

\begin{abstract}
Open-loop control strategies for legged robot locomotion have been investigated by many researchers because of the advantages in terms of simplicity and robustness, although the influence of control inputs to locomotion performance is not fully clarified. This paper investigates two of the most basic forms of control input, sinusoidal and pulsed signals, to be used in a class of hopping robot based on parallel elastic actuation. Our results show that a pulsed torque outperforms its sinusoidal counterpart with a lower energy expenditure. Moreover, the pulsed driving torque is capable of keeping the same energy efficiency while changing the forward hopping velocity, which is not possible with the sinusoidal driving torque. Such findings will help shape the future of robotics by achieving higher energy efficiencies within legged robots, while maintaining behavioural diversity.
\end{abstract}

Index Terms-open-loop control, energy efficiency, pulsed torque, sinusoidal torque, hopping.

\section{INTRODUCTION}

A LTHOUGH research shows that walking is inefficient when compared to wheels, swimming, and even flying [1], the locomotory versatility offered by legs allows animals to tread over different terrains. The superior stability and efficiency of animals, as opposed to legged machines, is ascribed to muscular properties [2], and the beneficial stability effects of such properties were partially mimicked in musculoskeletal robotic setups [3]. Energy efficient solutions for robotics have risen over the last years, ranging from passive walkers [4] to a combination of passive elements and actuators [5], [6], notably in theoretical work with (a) hopping and running of a spring-mass system [7] or by (b) the exploitation of natural frequencies to produce hopping motion [8]. Seeking to combine the broad spectrum of locomotion patterns suggested in the former with the simplicity and efficiency from the latter, the idea of parallel elastic actuation was adopted with an openloop sinusoidal actuation pattern to create a horizontal motion with a hopping robot [9]. In 2015, this same design and openloop control were upscaled and used to carry a $100 \mathrm{~kg}$ payload with unprecedented efficiency [10].

Open-loop control has demonstrated the potential to generate highly efficient locomotion when the elastic properties of the morphology are correctly exploited [11], [12], and hitherto this control method has been widely used in combination with sinusoidal control parameters [13], [14]. Owing to the cyclic nature of legged locomotion [15], sinusoidal and other continuously cyclical control variants (e.g. Buehler clock [16]) sought to mimic the patterns observed in nature and have always been preferred over pulsed control methods to avoid possible losses during impact with floor contact.

In this paper, we explore an alternative control method for a hopping robot and seek to improve its energy efficiency.
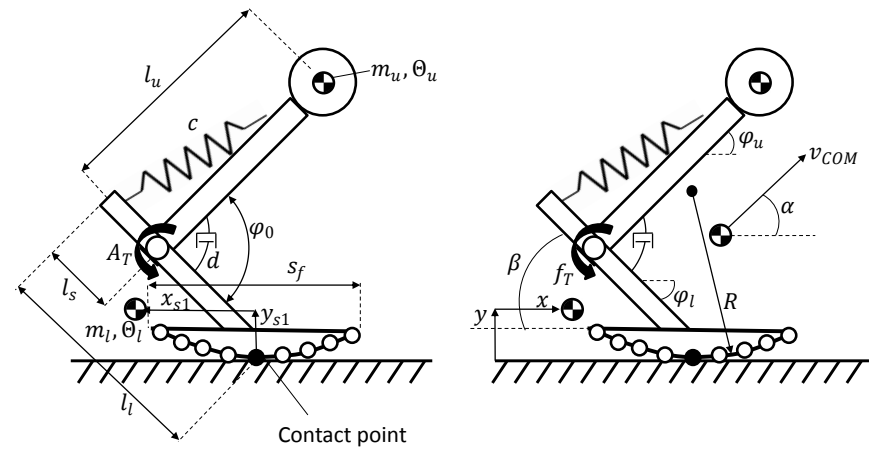

Fig. 1. Diagram with simulation model of the proposed robot. It hops with a curved feet, propelling its body forward and has a spring and an actuator arranged in parallel.

While our previous work [9] assailed exclusively the hopping efficiency with a sinusoidal driving pattern, in here we compare the previous pattern with a pulsed driving pattern through a fine-grained approach. The proposed control method impels the system to behave passively during the initial interaction with the floor and uses a torque surge to propel its body forward. Beyond the work from [9], we show that the pulsed torque solution outperforms the previous solution in energy efficiency. Although both control strategies have an intrinsic stability with observed phase locking, we show that the pulsed solution has lower energy losses during impacts and damping as it smoothly transitions from passive landing to active takeoff. Moreover, the pulsed driving torque offered a wide range of hopping speeds with little loss in energy efficiency. These findings will help future robotic applications create a nexus between legged systems and energy efficiency, and finally provide legged robots to be integrated into our lives.

We structured this paper as follows: In Section II, we introduce the simulation model, robot geometry and experimental method. In Section III, we present the results from simulations and experiments for the sinusoidal and pulsed driving methods. Section IV then discusses the results and their agreement with previous researches, while Section V contains a conclusion for this work.

\section{Model AND Methods}

The mathematical model adopted is illustrated in Fig. 1. All the relevant robot parameters for the following model description are listed in Table I together with their numerical values, measured from the real world robot. Due to the symmetry of the robot, the model can be reduced to a planar case. Two rigid bodies are linked to each other with a revolute 
TABLE I

PARAMETERS AND VARIABLES FOR THE HOPPING ROBOT.

\begin{tabular}{|c|c|c|}
\hline \multicolumn{3}{|c|}{ Constant Parameters } \\
\hline Symbol & Name & Value/Unit \\
\hline$c$ & Spring stiffness & $3022 \mathrm{~N} \mathrm{~m}^{-1}$ \\
\hline$d$ & Damping coefficient, knee joint & $0.084 \mathrm{~N} \mathrm{~s} \mathrm{~m}^{-1}$ \\
\hline$l_{l}$ & Lower leg length & $0.214 \mathrm{~m}$ \\
\hline$l_{u}$ & Upper leg length & $0.16 \mathrm{~m}$ \\
\hline$l_{s}$ & Moment arm length, spring mounting & $0.05 \mathrm{~m}$ \\
\hline$m_{l}$ & Lower body mass & $0.245 \mathrm{~kg}$ \\
\hline$m_{u}$ & Upper body mass & $0.355 \mathrm{~kg}$ \\
\hline$R$ & Foot radius & $0.3 \mathrm{~m}$ \\
\hline$s_{f}$ & Foot length & $0.2 \mathrm{~m}$ \\
\hline$\beta$ & Lower leg to foot angle & $1.1 \mathrm{rad}$ \\
\hline$x_{s 1}$ & Horizontal position of lower leg CoM & $-0.063 \mathrm{~m}$ \\
\hline$y_{s 1}$ & Vertical position of lower leg CoM & $0.045 \mathrm{~m}$ \\
\hline$\theta_{l}$ & Lower leg (plus foot) moment of inertia & $0.001433 \mathrm{~kg} \mathrm{~m}^{2}$ \\
\hline$\theta_{u}$ & Upper leg moment of inertia & $0.001055 \mathrm{~kg} \mathrm{~m}^{2}$ \\
\hline$\varphi_{0}$ & Knee angle for spring relaxed & $0.7854 \mathrm{rad}$ \\
\hline$r_{g}$ & Knee drive gear ratio & $155: 12$ \\
\hline$t_{c}$ & Motor torque constant & $0.131 \mathrm{Nm} \mathrm{A}^{-1}$ \\
\hline \multicolumn{3}{|c|}{ Minimal state coordinates } \\
\hline$x(t)$ & $\begin{array}{l}\text { Horizontal position of the lower leg } \\
\text { CoM }\end{array}$ & $\mathrm{m}$ \\
\hline$y(t)$ & Vertical position of the lower leg CoM & $\mathrm{m}$ \\
\hline$\varphi_{l}(t)$ & Lower leg angle & $\mathrm{rad}$ \\
\hline$\varphi_{u}(t)$ & Upper leg angle & $\mathrm{rad}$ \\
\hline \multicolumn{3}{|c|}{ Computed Variables } \\
\hline$T_{M}(t)$ & Motor torque function & $\mathrm{Nm}$ \\
\hline$P_{M}(t)$ & Motor power input function & $\mathrm{W}$ \\
\hline$\alpha(t)$ & $\begin{array}{l}\text { Take-off angle of the overall system } \\
\text { CoM }\end{array}$ & $\mathrm{rad}$ \\
\hline$\varphi(t)$ & Knee joint angle & $\mathrm{rad}$ \\
\hline$\omega_{T}(t)$ & Motor torque angular velocity & $\operatorname{rads}^{-1}$ \\
\hline
\end{tabular}

joint subject to linear damping with damping coefficient $d$. The system is sufficiently defined by the minimal coordinates

$$
\boldsymbol{q}=\left(x, y, \varphi_{l}, \varphi_{u}\right)^{T},
$$

with $x$ the horizontal coordinate of the lower body's centre of mass, $y$ its vertical coordinate, $\varphi_{l}$ its angle relative to the inertial coordinate frame, and $\varphi_{u}$ the angle of the upper rigid body relative to the inertial coordinate frame. A curved foot with radius $\mathrm{R}$ and length $s_{f}$ is firmly attached to the lower body with angle $\beta$. A linear spring is acting between the upper body centre of mass and the lower body. A time dependent motor torque $T_{M}$ is applied to the two bodies of the revolute joint. The torque is allowed to be any function of time, but within this work we will focus exclusively on sinusoidal and pulsed signals, illustrated in Fig. 2 . The sinusoidal function is described by the amplitude $A$ and the period $T_{s}$. The pulsed, square-wave signal is defined by the amplitude of the positive phase of the period $A_{h i}$, the negative phase of the period $A_{l o}$, the duty cycle $\lambda$, and the period $T_{p}$.

The equations of motion were derived using the projected Newton-Euler equation. This method uses the momentum and spin stated as a function of the generalised coordinates of the

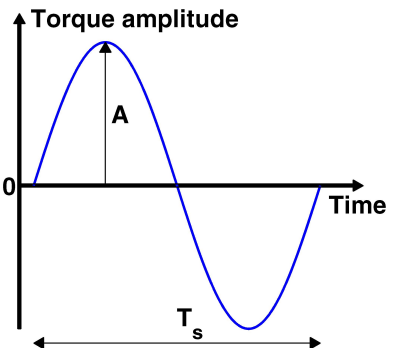

(a)

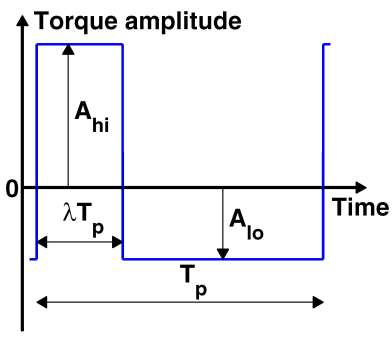

(b)
Fig. 2. The applied torque waveforms considered for this work. (a) a zeromean sinusoidal waveform and (b) a pulsed waveform.

individual rigid bodies in the system to calculate the inertial forces:

$$
0=\sum_{i=1}^{n}\left[\mathbf{J}_{S}^{T} \cdot \dot{\boldsymbol{p}}_{S}+\mathbf{J}_{R}^{T} \cdot \dot{\boldsymbol{N}}_{S}-\mathbf{J}_{Q}^{T} \cdot \boldsymbol{F}_{E}-\mathbf{J}_{R}^{T} \cdot \boldsymbol{M}_{E}\right]_{i}
$$

with $n$ the number of rigid bodies in the system, $\mathbf{J}_{S}$ the Jacobian of the centre of mass $S$ of body $i, \dot{p}_{S}$ the first derivative in time of the momentum of body $i$ with respect to the centre of mass $S, \mathbf{J}_{\mathbf{R}}$ the rotational Jacobian of body $i, \dot{N}_{S}$ the first derivative in time of the spin of body $i$ with respect to the centre of mass $S, \mathbf{J}_{Q}$ the Jacobian at the point of attack of the external forces acting on the body $i$, $\boldsymbol{F}_{E}$ the external force acting on the body $i$, and $\boldsymbol{M}_{E}$ the external moment acting on the body $i . \boldsymbol{F}_{E}$ is in our case the gravitational force acting on the centre of mass of the upper and lower rigid leg, whereas $M_{E}$ is the motor torque applied to the joint in the system. The resulting equation can be restructured to the form

$$
\mathbf{M} \dot{\boldsymbol{u}}+\boldsymbol{h}(\boldsymbol{u}, \boldsymbol{q})=0
$$

with $\mathbf{M}$ the mass matrix containing all the coefficients of the second derivatives in equation 2 , and $\boldsymbol{h}$ the vector of gyroscopic accelerations containing all the remaining terms including motor torque and gravity, with $\boldsymbol{u}=\dot{\boldsymbol{q}}$ the vector of generalised velocities.

Due to the impulsive nature of the considered system, the nonlinear equations are reformulated into an equality of measures

$$
\mathbf{M d} \boldsymbol{u}+\boldsymbol{h}(\boldsymbol{u}, \boldsymbol{q}) \mathrm{d} t=\mathrm{d} \boldsymbol{R},
$$

with $\mathrm{d} \boldsymbol{R}$ the impulsive contact forces. During phases with no collision, the equations of motion can simply be integrated numerically using a midpoint rule [17], neglecting the vector $\mathrm{dR}$. As soon as a collision event occurs, that is, when the foot touches down on the ground, the ground contact forces are being calculated by a Newtonian kinematic impact law with a coefficient of restitution of zero (i.e. plastic impact), defined by

$$
\gamma_{N}^{+}=0
$$

with $\gamma_{N}^{+}=0$ the velocity normal to the impact point right after the impact event, and a Coulomb friction law described by

$$
F_{T} \in \mu F_{N} \operatorname{Sgn}\left(\gamma_{T}\right),
$$


with $F_{T}$ the friction force, $\mu$ the coefficient of kinetic friction, $F_{N}$ the normal force on the contact point, Sgn the set-valued signum function, and $\gamma_{T}$ the tangential velocity of the contact point. Note that the equality sign is avoided due to the setvalued nature of the Sgn function, and the set membership sign $\in$ is applied. The task of finding the contact forces is reformulated to a linear complementarity problem (LCP), as presented in [18]. The solution of the LCP, found by enumerative methods, defines the contact forces between the foot and the ground. These are fed back to the integration scheme to proceed as usual.

The model has previously been applied and found to lead to accurate trajectory predictions of the robot [9]. However, the limitations of the model need to be considered. First, the motor and drive belt dynamics are neglected and the torque is assumed to build up instantaneously by the motor. Second, the collisions are modelled with a Newtonian kinematic impact law and a Coulomb friction law, which makes sense only if the materials participating in the collision are nonelastic, and their tangential forces do not depend on the contacting surface area. Furthermore, the linear damping assumption in the joint might further deteriorate the real world results from the experiment. Lastly, a rigid body assumption neglects all the internal wave propagations in the material, especially due to hard impacts.

\section{A. Simulation methods}

The mathematical model previoulsy presented was used to predict the locomotion gait behaviour for the robot for a duration of five seconds from a standing start. For each of the torque functions presented in Fig. 2 a suitable parameter space was chosen for detailed investigation based on knowledge of the resonant frequency of the mechanical system and the torque capabilities of the chosen drive motor. These parameter spaces are presented alongside the simulated results. For this work all mechanical parameters of the robot are kept constant, and solely variations in the actuation torque are considered. The existing lack of sensors means that no pre-established timing for the exertion of torques exists, and thus any attraction between the actuation and locomotion is a product of the relationship between the body, the controller and the environment.

Due to the non-linearity of the system, formal stability definitions are difficult to define and the stability indicators used in [9] were adapted as follows:

1) A parameter set yielding locomotion for five seconds after accelerating from rest without falling over is considered successful.

2) The standard deviation for the take-off angle of the robot's centre of mass $\alpha$ is used as an indicator of stability. A lower standard deviation corresponds to improved system stability. The standard deviation was taken over all hops except the first two to enable consideration of only the steady state hopping behaviour.

This definition of stability mimics those of Lyapunov stability theory, such as that presented by $[19, \mathrm{p} .48]$ :

$$
\forall R>0, \exists r>0,\|\mathbf{x}(0)\|<r \Rightarrow \forall t \geq 0,\|\mathbf{x}(t)\|<R
$$

which states that a stable solution $\mathbf{x}(t)$, represented by a subset of state variables, must remain bounded to a region $R$ near the equilibrium, for an initial condition $\mathbf{x}(0)$ bounded by $\mathbf{r}$.

For quantification of the locomotion efficiency of the system the measure proposed in [1] has been used, defining the cost of transport (CoT):

$$
\operatorname{CoT}=\frac{E_{i}}{W d}
$$

where $E_{i}$ is the net energy supplied to the system during locomotion, $W$ is the weight of the system, and $d$ is the distance translated horizontally. The energy input considers mechanical energy input and losses only since the simulation neglects all motor and actuation dynamics.

Although these two quantifiers for stability and efficiency are suggested, this work will focus on the latter, and stability will be only considered to validate our results as feasible. We acknowledge that stability is also very important, and we have approached this topic with similar hopping mechanism in previous papers [8], [9], [10].

\section{B. Experimental methods}

The objective of our experiments was to validate the simulated behaviour, particularly the robot's hopping gaits and the CoT for the system. Additionally, we sought to verify the modelling assumptions discussed previously, particularly the assumption of negligible dynamics for the knee joint actuation system. It was also desired to obtain suitable values of parameters $\mu$ and $d$ (the ground friction and the knee damping) to yield comparable simulation results. This was achieved by tuning these parameters such that the steady-state gaits of the robot from simulation and real-life matched, as was done for a previous work [9].

The energy supplied to the robot motor was measured with a Two-Wattmeter circuit, and the architecture is detailed in [20]. The energy input to the motor was used for the evaluation of the cost of transport, serving as the input $E_{i}$ from Eqn. 8.

An Optitrack optical motion tracking system was used to record the 3D motion of the experiments. Reflective spherical markers placed on the robot were used to define rigid bodies to be tracked during locomotion. The gaits from simulation and experiments were compared by consideration of the trajectories of points on the robot. The trajectories of the knee joint, the motor axis centre, and the mid-point of the lower leg tube were used for convenience. The simulation was tuned to better represent the behaviour observed during preliminary experiments. While adjusting the parameters, no changes were needed for the frictional coefficient and only minimal change to the damping factor was required to correlate simulation/experiment. Setting the hinge joint damping factor in this way accounts for the combined losses from joint friction, motor losses, and transmission losses in a single parameter.

To validate the simulated behaviour, a robot was constructed and is shown in Fig. 3. The upper and lower legs are made from carbon fibre tubes. A pair of MDF feet spaced $0.2 \mathrm{~m}$ apart are used to provide sagittal plane stability to the otherwise planar structure considered in simulation. The hinge joint 


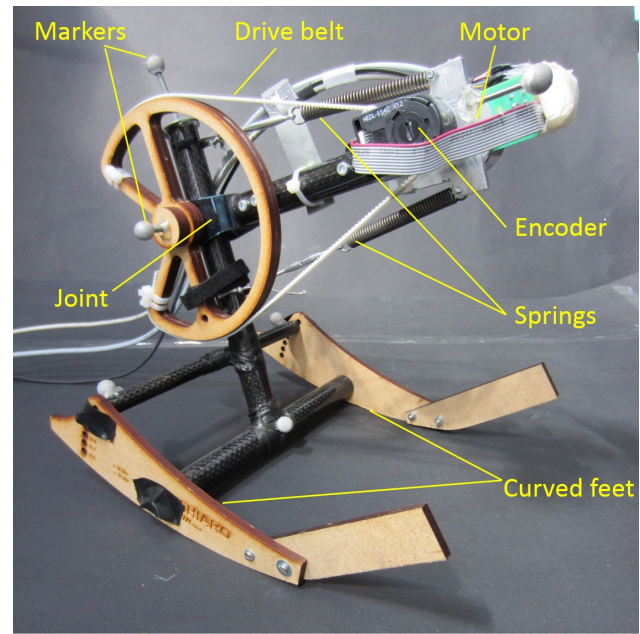

Fig. 3. The experimental setup with the hopping robot. The straight extensions to the front end of the foot were added to protect the motor assembly from damage during testing by minimising the risk of the robot falling over fully, and do not contact the ground to impact the robot gait during normal operation.

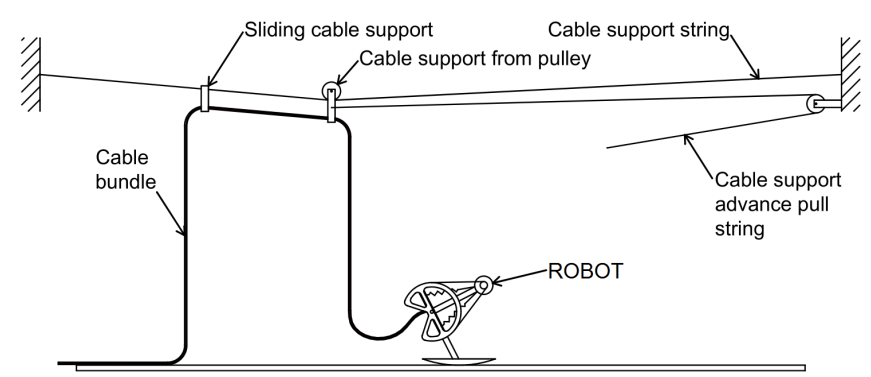

Fig. 4. A schematic of the test rig used to consistently support the power and control cables connected to the robot during testing.

connecting the upper and lower leg uses ball bearings to minimise frictional losses and these are supported using 3Dprinted components. The joint is actuated through a toothedbelt reduction drive by a Maxon EC45 flat brushless-DC motor capable of supplying $70 \mathrm{~W}$ of power. A Maxon EPOS 70/10 positioning controller was used to regulate the motor torque under a Matlab algorithm. The motor controller was tethered to avoid its substantial mass affecting the robot. The pushpull spring used in simulation was realised with a pair of opposing pull-springs with half the stiffness. A necessary practical consideration during testing is the support of the cables used to connect the motor to the motor controllerthe mass and stiffness of the cable bundle may easily cripple the locomotion of the robot unless care is taken to support it during the test run. To minimise the effect of the cable bundle on the robot dynamics the cables were slung from a support which translated with the robot under the control of the operator as shown in Fig. 4.

\section{RESULTS}

The simulation and experimental results are presented in this section. Sinusoidal torques (which were previously used in [9])are considered first as a performance standard against which the performance from pulsed torque inputs is compared.

\section{A. Simulations with sinusoidal driving torque}

For the sinusoidal torque waveform variations in both the waveform period $T_{s}$ and the amplitude $A$ were considered. A parameter space comprised of waveform frequencies from $2.5 \mathrm{~Hz}$ to $4.5 \mathrm{~Hz}$ and knee joint torque amplitudes from $0.1 \mathrm{Nm}$ to $1.5 \mathrm{Nm}$ were considered. The system has a peak resonant response at a frequency of $4.5 \mathrm{~Hz}$ but substantially lower responses at frequencies above this. The majority of the frequencies considered hence correspond to below-resonance excitation of the system.

A scatter plot of the CoT with respect to locomotion speed for this parameter space is shown in Fig. 5a, in which the points are additionally colour coded according to the standard deviation of the take-off angle $\alpha$ to give an indication of the stability of the underlying gaits. Points for which the standard deviation of $\alpha(\sigma(\alpha))$ is less than five degrees are coloured black to indicate that these gaits are very stable. Runs for which $\sigma(\alpha)$ is larger than five degrees or for which no hopping was observed (the robot instead shuffling forwards whilst always maintaining ground contact) have points which are coloured grey.

The lowest value for CoT within Fig. 5a indicates that the system moves most efficiently with a sinusoidal torque input for a forward locomotion speed of $0.2 \mathrm{~m} \mathrm{~s}^{-1}$. This solution has a CoT of 0.83 and requires a waveform frequency of $4.3 \mathrm{~Hz}$ and a torque amplitude of $0.55 \mathrm{Nm}$. An efficiency penalty is seen for locomotion speeds either side of this most efficient speed. From the work of [1] and later [2], a biological system of comparable mass to that used in this work would be expected to have a minimal Total CoT in excess of 2 (no data on metabolic costs are provided though), which indicates that the system considered in this work is predicted to move efficiently for its mass after losses are accounted for. It is relevant to note that the most efficient solutions are generally those which are the most stable. The converse is not necessarily the case: highly stable solutions are not always efficient.

Fig. 5b considers the structure of the data presented in Fig. 5a by connecting solutions of equal frequency and adjacent torque amplitudes with lines. Increases in the amplitude (A) correspond to an increase in forward speed as indicated by the arrow on the plot. From this figure, it is apparent that the most efficient solution is not constrained by the choice of parameter. Conversely, it appears that this limit is due to a more fundamental aspect of the dynamics of the system when driven using a sinusoidal input, as shown by the clustering of lines of constant frequency around the most efficient speed for frequencies around $4.3 \mathrm{~Hz}$. For frequencies below $3.5 \mathrm{~Hz}$ the CoT decreases with increasing locomotion speed until stability is lost and the robot falls. Hence, a lower CoT should be enforced by continuously changing amplitude and frequency for different forward locomotion speeds.

Phase plots for the system state variables for the most efficient simulated solutions with forward locomotion speeds of $0.2 \mathrm{~m} \mathrm{~s}^{-1}$ and $0.41 \mathrm{~m} \mathrm{~s}^{-1}$ are shown in Fig. 6. It is apparent from Fig. $6 \mathrm{~b}$ that this higher speed solution (which is grouped with those which are unstable in Fig. 5a) is an example of a 


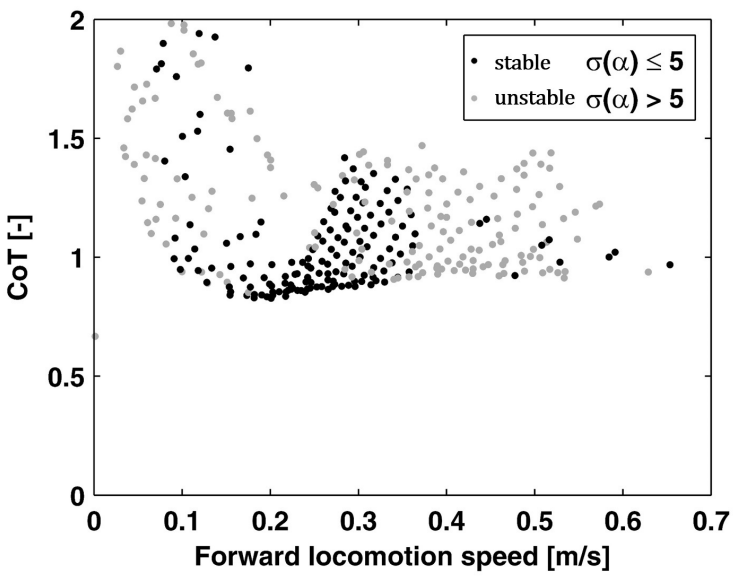

(a)

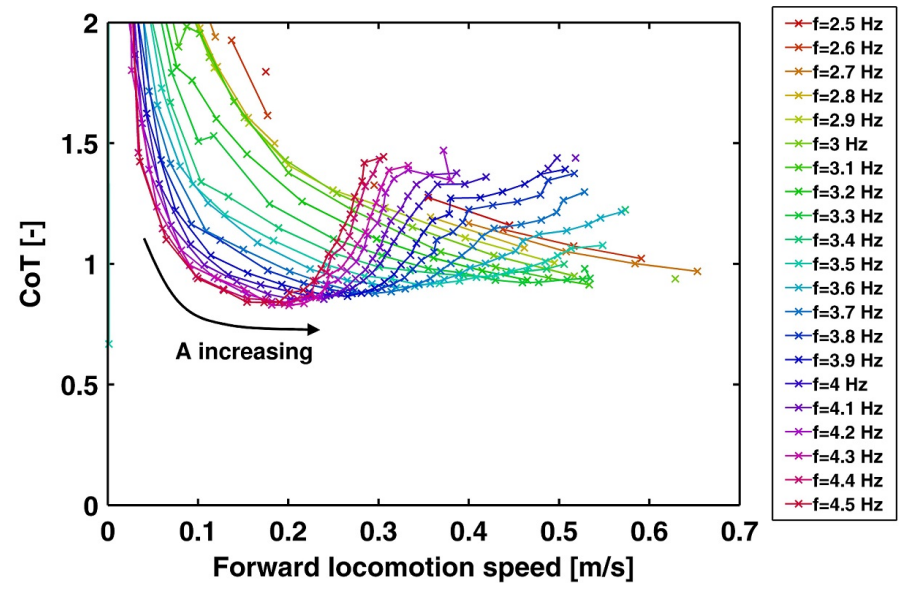

(b)

Fig. 5. Simulated CoT and speed with a sinusoidal waveform, showing (a) stability information and (b) parameter space navigation information.

\section{$0.2[\mathrm{~m} / \mathrm{s}]$}
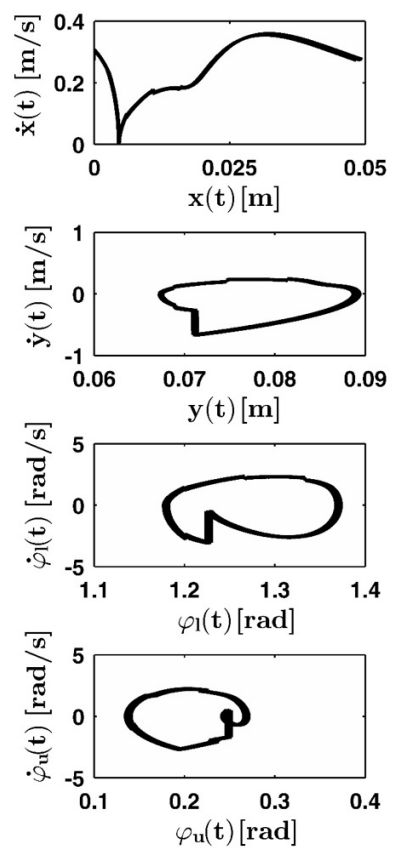

(a) $0.41[\mathrm{~m} / \mathrm{s}]$
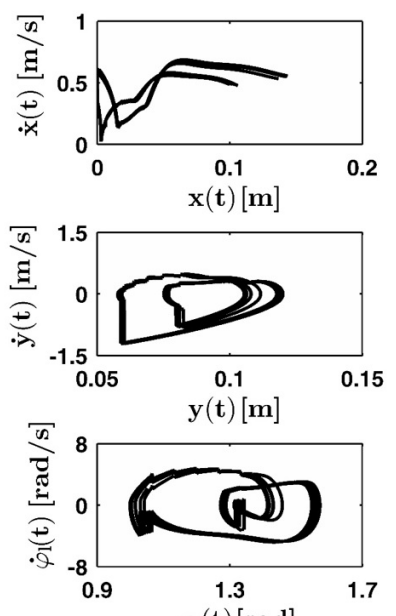

$\varphi_{1}(\mathrm{t})[\mathrm{rad}]$

(b)

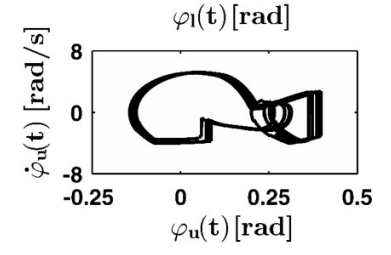

Fig. 6. Phase plots for the system state variables for the last ten hops of the most efficient simulated solutions using a sinusoidal driving torque for forward locomotion speeds of (a) $0.2 \mathrm{~m} \mathrm{~s}^{-1}$ and (b) $0.41 \mathrm{~m} \mathrm{~s}^{-1}$. The solution for (a) requires a driving waveform frequency of $4.3 \mathrm{~Hz}$ and torque amplitude of $0.55 \mathrm{~N} \mathrm{~m}$ whilst (b) requires a frequency of $3.5 \mathrm{~Hz}$ and a torque amplitude of $1 \mathrm{Nm}$.

period-2 gait, deemed unstable by our chosen stability criteria. Consideration of the phase plots for many solutions using sinusoidal driving torques has revealed that the occurrence of such period-2 gaits are common with this control input.

\section{B. Simulations with pulsed driving torque}

A pulsed torque waveform, as shown in Fig. 2b, was considered as an alternative to the sinusoidal torque input.
A parameter space comprised of waveform frequencies from $3 \mathrm{~Hz}$ to $4.6 \mathrm{~Hz}$, duty cycles from $5 \%$ to $50 \%$, and torque amplitudes varying from $0 \mathrm{Nm}$ to $1.5 \mathrm{Nm}$ was initially considered. Duty cycles in excess of $50 \%$ and frequencies below $3 \mathrm{~Hz}$ were not considered for investigation as they did not yield stable locomotion behaviour.

During simulations we noted that the most efficient solutions were those which used duty cycles below $18 \%$, and we also observed that for best efficiency it was desirable to set the negative portion of the torque waveform to zero (by setting parameter $A_{l o}$ to zero). Such actuation pushed the robot upward prior to the transition from the stance phase to the flight phase, and the motor was fully back-drivable when no torque was applied, further increasing efficiency.

A scatter plot of the CoT as a function of forward locomotion speed for the pulsed method is shown in Fig. 7a, with the points colour coded for purposes of stability indication analogously to the sinusoidal case presented in Fig. 5a. This figure indicates that when a pulsed torque is used the system demonstrates a substantially lower mechanical CoT (below $0.5)$. Furthermore, simulation speeds ranging from $0.1 \mathrm{~m} \mathrm{~s}^{-1}$ to $0.65 \mathrm{~m} \mathrm{~s}^{-1}$ presented a minimal efficiency penalty for variation of the locomotion speed. It can be observed that these solutions are generally very stable by our stability criteria.

The variations of the CoT with locomotion speed for a waveform frequency of $3.2 \mathrm{~Hz}$ is presented in Fig. 7b. As in Fig. $5 \mathrm{~b}$ for the sinusoidal case, data points with identical duty cycle value and adjacent positive torque amplitude values $\left(A_{h i}\right.$ values) in the parameter space are connected with straight lines. While in Fig. 5b all data points from Fig. 5a were represented, the proximity of datapoints in Fig. 7a led Fig. 7b to a more simplified representation to enhance understanding. As indicated by the arrow on the plot, increasing torque amplitudes increase the locomotion speed. The end of the line for a duty cycle of $5 \%$ is circled to indicate that this run uses the largest amplitude value in the parameter space, indicating that the solution explored the entire parameter space. The occurrence of the best solutions during small duty cycles hints 


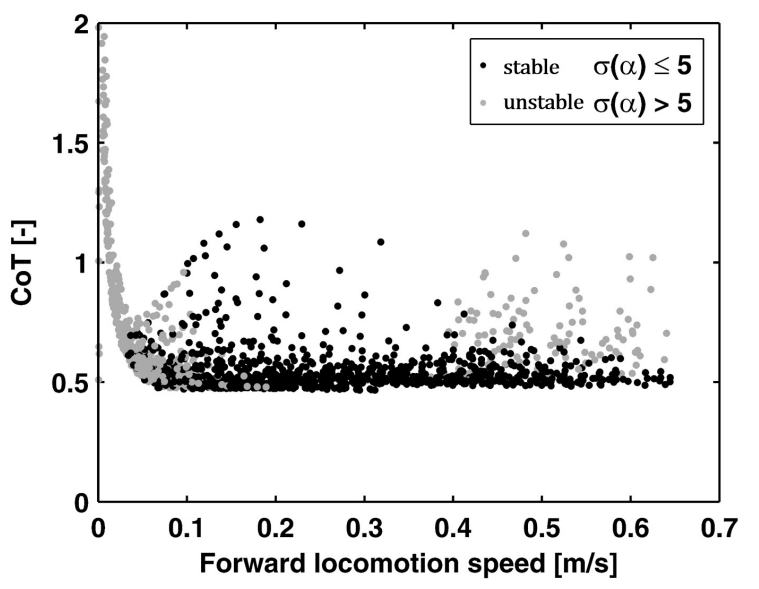

(a)

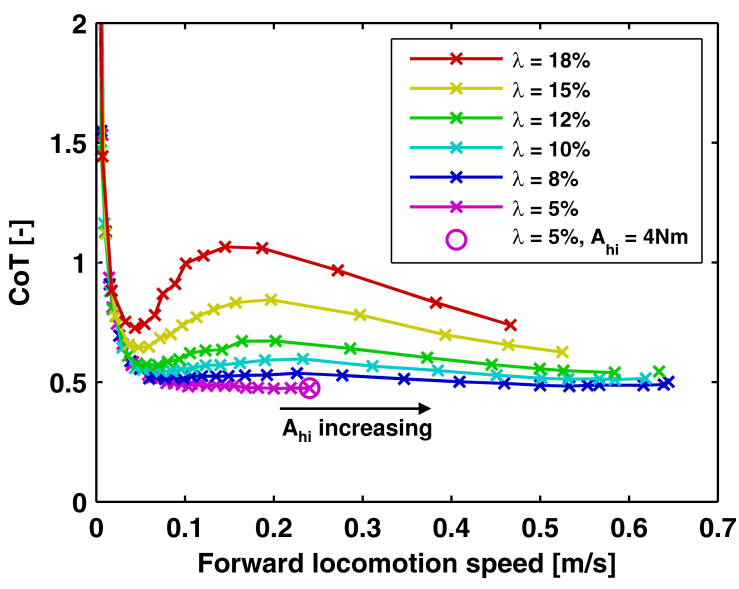

(b)

Fig. 7. Simulated CoT and speed for pulsed waveform with $A_{l o}=0$. Showing (a) stability information for the whole parameter space and (b) parameter space structure for a frequency of $3.2 \mathrm{~Hz}$, permitting positive torque amplitudes up to $4 \mathrm{Nm}$. Additional trends from (a) were omitted while plotting (b) to avoid clutter and facilitate understanding.

to the superiority of impulsive forces, where the continuity in this minimal CoT was kept in Fig. 7a for locomotion speeds near $0.3 \mathrm{~m} \mathrm{~s}^{-1}$. Fig. $7 \mathrm{~b}$ demonstrates that small duty cycles are optimal for both the locomotion efficiency and forward locomotion speed variation. An additional desirable property of the pulsed torque solutions is that for a constant frequency and duty cycle, open-loop variations in the amplitude of the torque pulse may be used to vary the forward locomotion speed in the optimal regime, providing that sufficiently large torque pulses may be applied.

Phase plots for the state variables for the most efficient pulsed torque solutions at speeds of $0.2 \mathrm{~m} \mathrm{~s}^{-1}$ and $0.41 \mathrm{~m} \mathrm{~s}^{-1}$ (to compare with those for a sinusoidal drive torque in Fig. 6) are given in Fig. 8. It is apparent that these gaits only show minor indications of period-2 gaits, though are somewhat less consistent between hops than the previously presented sinusoidal solutions. This is attributed to the application of larger control inputs to the system by open-loop methodssmall differences between hops are now more amplified than previously. The larger torques do not have a destabilising effect on the system however.

The increased maximal efficiency of the pulsed torque solutions in comparison to sinusoidal ones is demonstrated in Fig. 9 which shows the lower bound on the CoT of the solutions from the simulated parameter space for sinusoidal torque waveforms alongside two of the most efficientpulsed torque solution sets. The substantial efficiency improvement offered by the pulsed torque waveform, which reduced the CoT from 0.8 , to 0.5 , is clear and highly desirable.

It is of interest to note that this efficiency gain is achieved by applying large control inputs, usually two or three times the torque amplitude from the sinusoidal methods, for brief duration. This stands in contrast to our previous work with hopping locomotion, which has sought to exploit mechanical resonance using a sinusoidal torque of minimal amplitude [9].

Within Figs. 10 and 11 we draw a comparison between the torque, angular velocity and power for two different forward
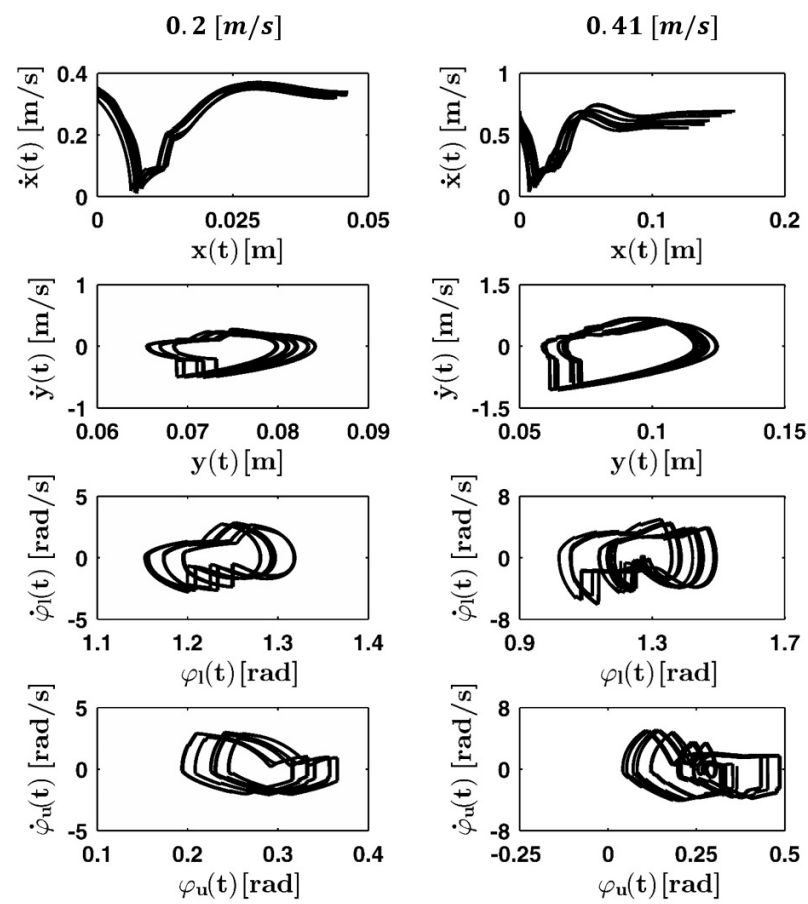

(a)

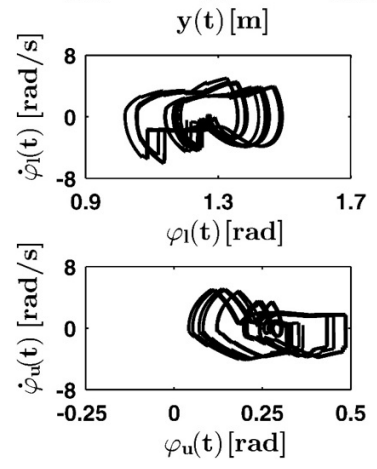

(b)

Fig. 8. Phase plots for system state variables for the last ten hops of the most efficient simulated solutions using a pulsed driving torque for forward locomotion speeds of (a) $0.2 \mathrm{~m} \mathrm{~s}^{-1}$ and (b) $0.41 \mathrm{~m} \mathrm{~s}^{-1}$. The solution for (a) requires a pulsed waveform of frequency $4.6 \mathrm{~Hz}$, positive torque amplitude of $3.6 \mathrm{~N} \mathrm{~m}$ and duty cycle of $5 \%$, whilst that of (b) requires a frequency $3.2 \mathrm{~Hz}$, positive torque amplitude of $2.9 \mathrm{Nm}$ and duty cycle of $8 \%$. Both solutions set the negative torque pulse amplitude to zero.

velocities $\left(0.2 \mathrm{~m} \mathrm{~s}^{-1}\right.$ and $\left.0.41 \mathrm{~m} \mathrm{~s}^{-1}\right)$ and the two aforementioned control methods. Within Fig. 10 we can observe the occurrence of period-2 gaits during higher forward velocities, and positive work is depicted in green while negative work is shown in red. During the pulsed driving method, depicted 


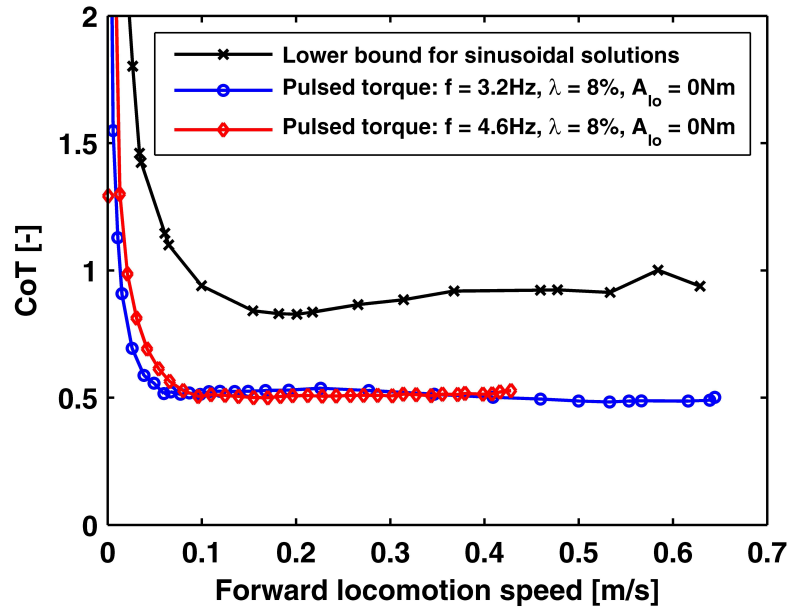

Fig. 9. The lower bound on the simulated CoT speed relation for a sinusoidal torque waveform compared to simulated solutions for a pulsed waveform with duty cycle of $8 \%$ and negative torque pulse amplitudes set to zero for frequencies of $3.2 \mathrm{~Hz}$ and $4.6 \mathrm{~Hz}$, allowing positive torque amplitudes up to $4 \mathrm{Nm}$.
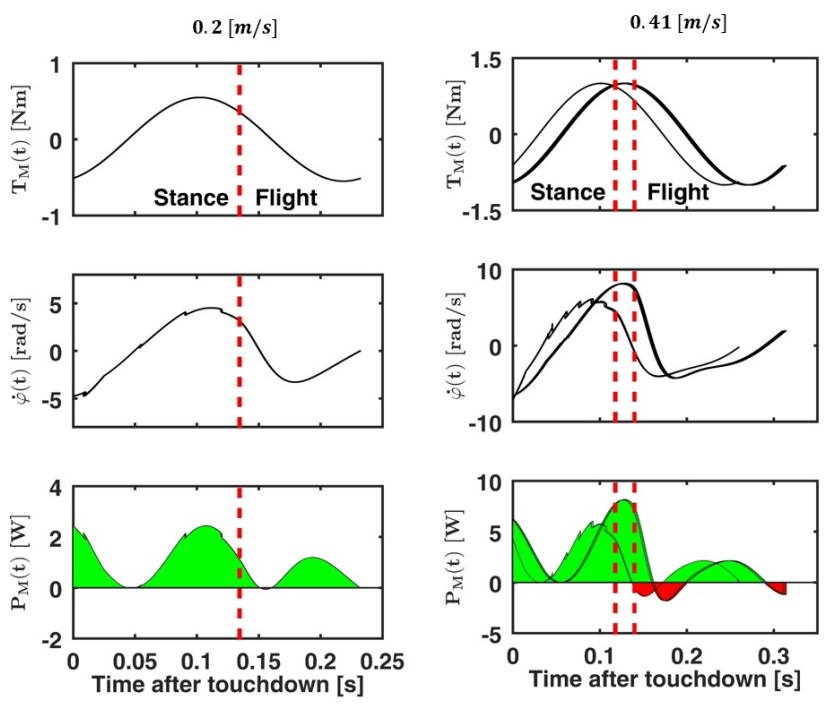

(a)

(b)

Fig. 10. Comparison of knee joint behaviour for the last five simulated hops of the most efficient sinusoidal torque solutions at locomotion speeds of (a) $0.2 \mathrm{~m} \mathrm{~s}^{-1}$ and (b) $0.41 \mathrm{~m} \mathrm{~s}^{-1}$. The red dotted vertical lines denote the transitions of the hops from the stance phase into the flight phase of motion.

in Fig. 11, a surge of torque occurs and affects the knee joint angle, with no other torque input provided besides. The sinusoidal driving method (Fig. 10) shows a detrimental performance during higher speeds, where the motor acts in the opposite direction of the movement, which is a role ascribed to the springs during the pulsed driving method. This extra burden on the motor accounts for energy losses on the sinusoidal method.

Fig. 12 presents the energy losses of the robot categorised into impact losses, frictional losses (in ground contact interactions) and damping losses from the knee joint for two locomotion speeds for each of the torque waveforms considered in this work. Although both cases had equivalent frictional losses,
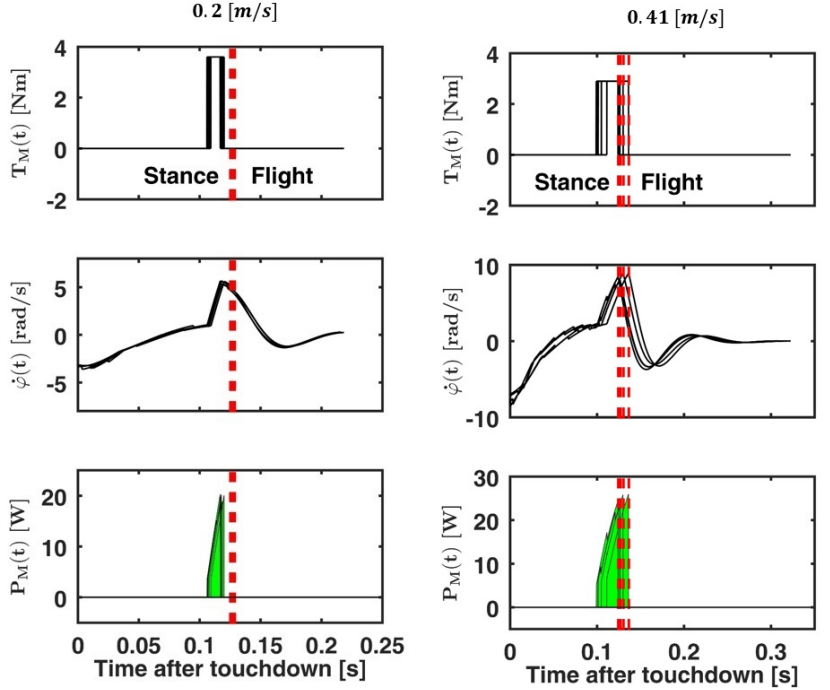

(a)

(b)

Fig. 11. Comparison of knee joint behaviour for the last five simulated hops of the most efficient pulsed torque solutions at locomotion speeds of (a) $0.2 \mathrm{~m} \mathrm{~s}^{-1}$ and (b) $0.41 \mathrm{~m} \mathrm{~s}^{-1}$. The red dotted vertical lines denote the transitions of the hops from the stance phase into the flight phase of motion.

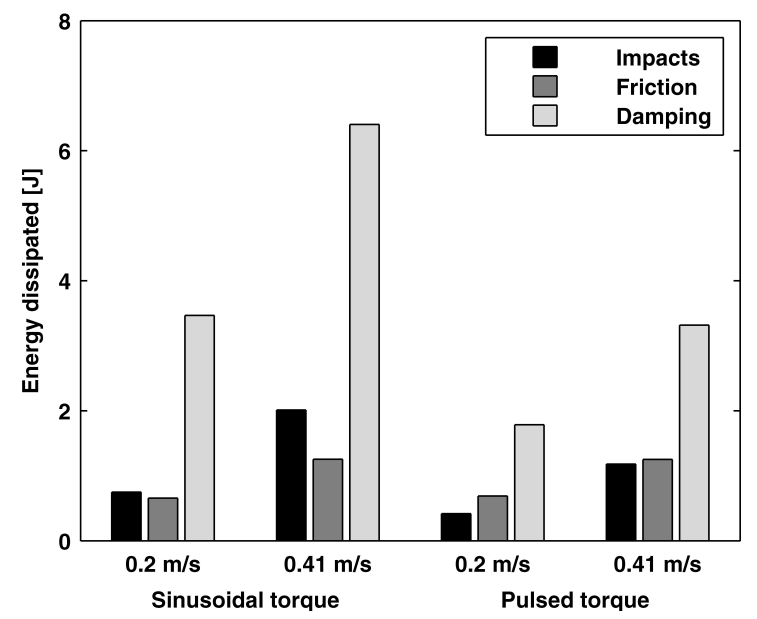

Fig. 12. Sources and magnitudes of energy loss during a five second simulation run for sinusoidal and pulsed torque solutions with locomotion speeds of $0.2 \mathrm{~m} \mathrm{~s}^{-1}$ and $0.41 \mathrm{~m} \mathrm{~s}^{-1}$. Ground contact losses combines dissipation from both foot sliding and ground impacts.

the pulsed torque method vastly outperforms the sinusoidal torque method when losses due to impact and damping are considered, with the pulsed case decreasing the losses by $46 \%$ in comparison to the sinusoidal case.

\section{Experiments}

Experiments were conducted for both sinusoidal and pulsed driving torques. In Fig. 13 we show motion capture data from experimental trials for the sinusoidal and pulsed driving methods, and both cases behave similarly. A clear trend for both cases consists in an initial adjusting phase, a steady-state phase, and the end of the experiment. Snapshots of the robot hopping are shown in Fig. 13c. 


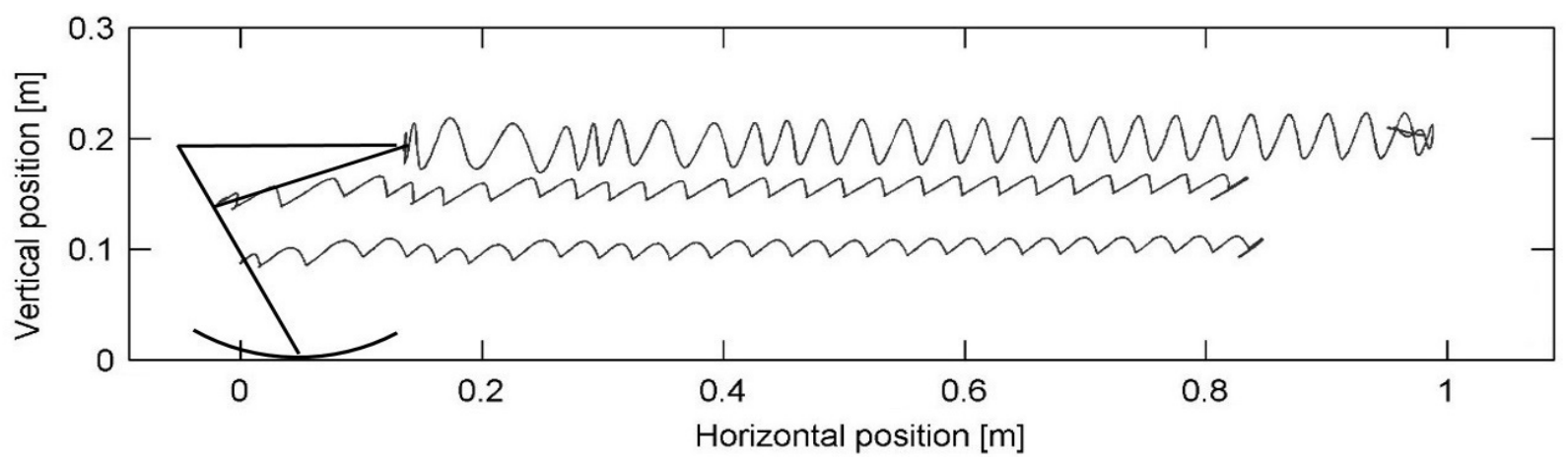

(a) Trajectories from motor, joint and leg with the sinusoidal input, frequency $4.5 \mathrm{~Hz}$, and amplitude $0.25 \mathrm{~A}$.

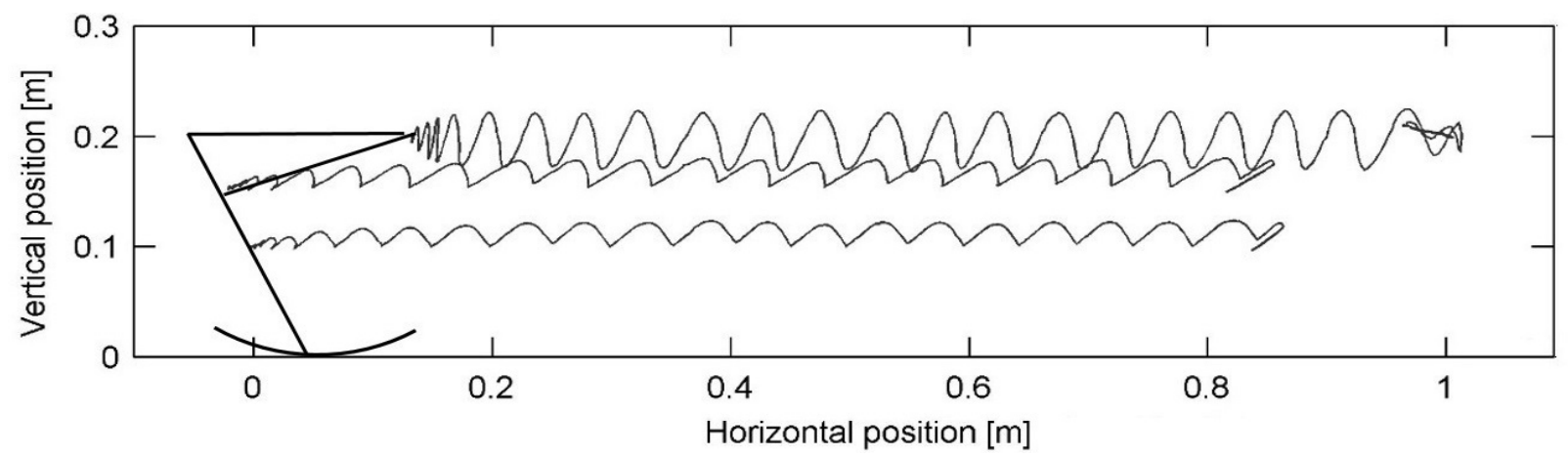

(b) Trajectories from motor, joint and leg with the pulsed input, frequency $4.1 \mathrm{~Hz}$, torque $1.2 \mathrm{~N} \mathrm{~m}$, and duty cycle of $10 \%$.
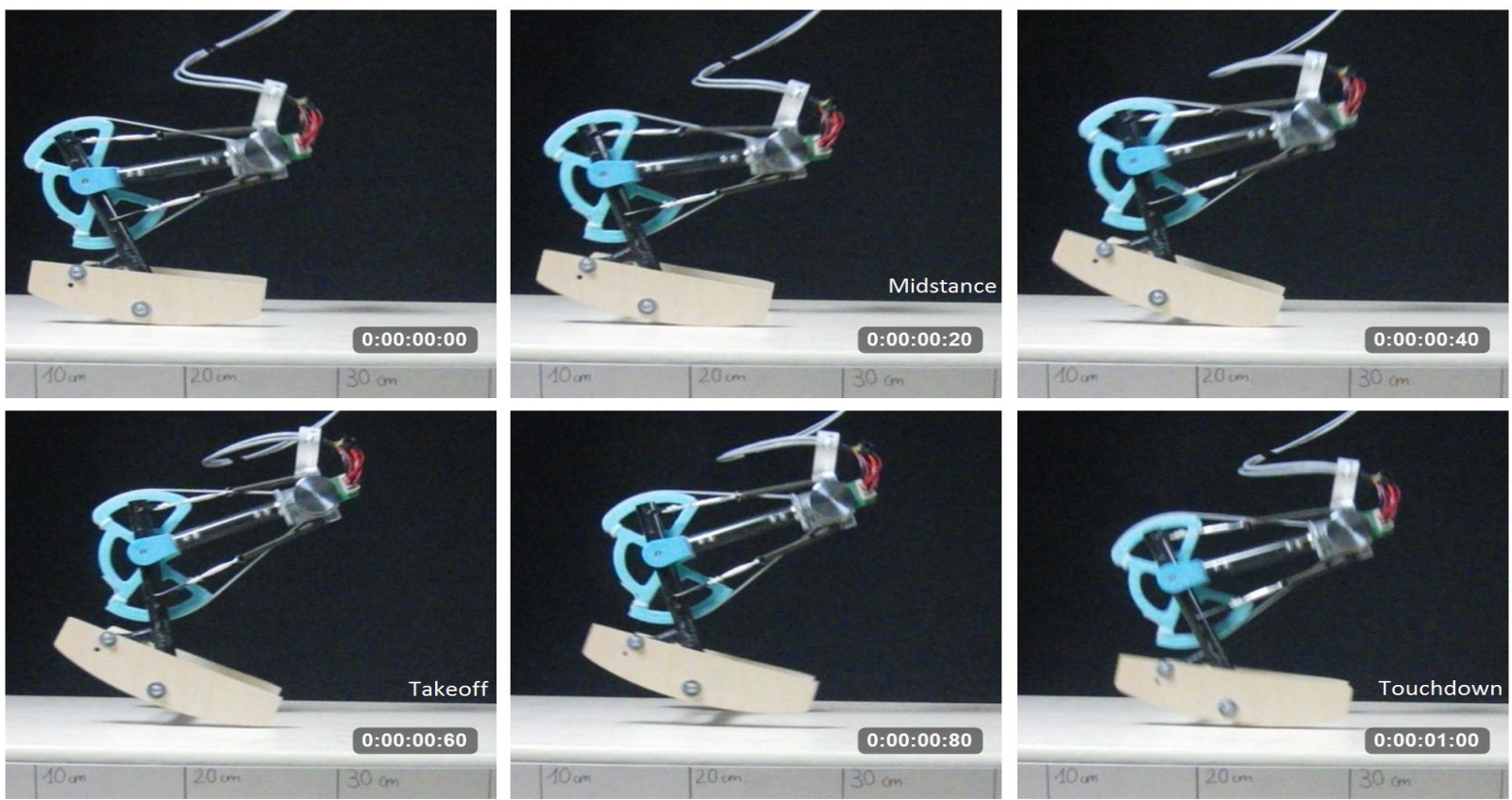

(c) Snapshots taken from the robot while hopping. As the robot inclines its body forward, the motor produces a torque to generate a diagonal thrust, as seen at $0.6 \mathrm{~s}$. The swing phase lasts from this lack of contact until touchdown, at $1 \mathrm{~s}$, preparing the robot for another hop.

Fig. 13. Motion capture data from experiments with sinusoid (a) and pulsed (b) torques depicting how locomotion converges to a steady state after a start-up phase. In (c) one steady state hopping cycle is illustrated.

Measurements were taken to create plots of the Total CoT as a function of locomotion speed for both experiments, analogously to Fig. 5b and Fig. 7b. The plot for a sinusoidal torque is given in gray within Fig. 14 and a minimal cost of transport of 0.8 was obtained, closely agreeing with simulation results. Within our experiments, locomotion speeds higher than $0.3 \mathrm{~m} \mathrm{~s}^{-1}$ were not possible for both control methods The solutions for frequencies of $4.1 \mathrm{~Hz}$ and $4.2 \mathrm{~Hz}$ and duty cycles of $10 \%$ for the negative pulse amplitude held at zero are depicted in black within Fig. 14. Although an agreement between experiments and simulations can be seen, in some occasions an increase in the target pulse amplitude to the 


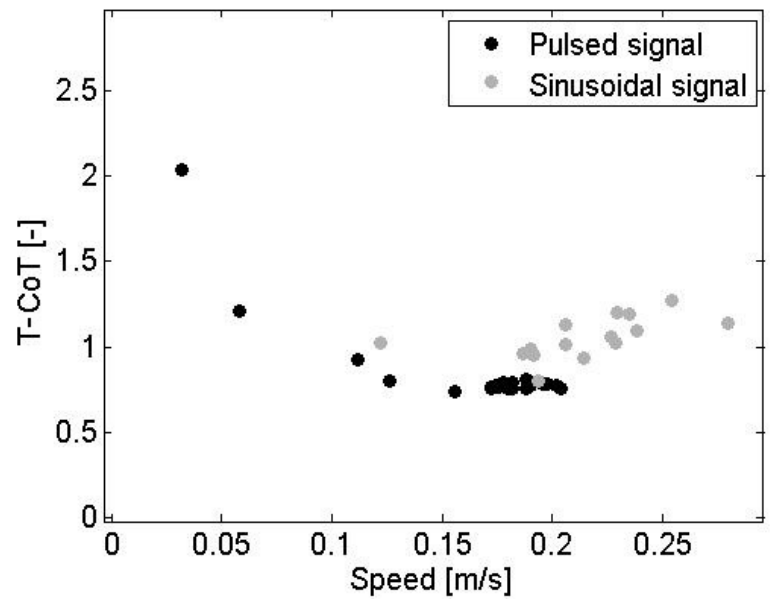

Fig. 14. Experimental relationship between the Total CoT and forward speed for sinusoidal and pulsed cases. Lower forward velocities were possible with pulsed control methods, and an efficient transport is always visible for these cases when compared to sinusoidal cases.

motor controller no longer increased the locomotion speed as expected, as shown by the clustering of the data points for speeds below $0.2 \mathrm{~m} \mathrm{~s}^{-1}$. The pulsed driving torque results presented a lower efficiency than predicted by simulation, but these were still better than the results for the sinusoidal method (0.72 vs 0.8 ).

As highlighted in Section II, the simulation of the system simplifies the dynamics of the driving torque to the knee joint. Though this assumption appears to be satisfactory in the case of sinusoidal driving torques, the discrepancy between the results of simulations and experiments would suggest that this simplification impacts negatively the pulsed case. We argue that, in addition to the simulated dynamics, the experiments have an extra rotational inertia to which the driving torque is applied (the motor armature). Since the belt has longitudinal compliance, these unaccounted model differences act as a lowpass filter and smoothen high frequency torque inputs (pulsed waveform).

A further source of discrepancy between the simulated and real-world results stems from the current limiter found at the motor controller. The target and actual torques applied to the motor armature (scaled by the drive belt reduction ratio) for two pulsed torque solutions are presented in Fig. 15. It is apparent that for the solution with a target torque pulse amplitude of $1.8 \mathrm{~N} \mathrm{~m}$ (Fig. 15b) the actual torque never reaches the target, but is generally limited to $1.4 \mathrm{Nm}$ or less, and for the solution with a target torque pulse amplitude of $1.2 \mathrm{~N} \mathrm{~m}$ (Fig. 15a) the torque is not reliably regulated to the target level. This maximum rate of change of torque is influenced by the current limitation and is responsible for the speed limit observed for pulsed torques within Fig. 14.

The stability of the pulsed torque driving method is proven with a phase plot, with recurring translational and rotational parameters, as seen in Fig 16. Both pulsed and sinusoidal cases had similar patterns for successful cases, with ten hops each, whilst unsuccessful cases resulted in collapsing or imobility. Finally, Fig. 14 shows how experimental results for pulsed and

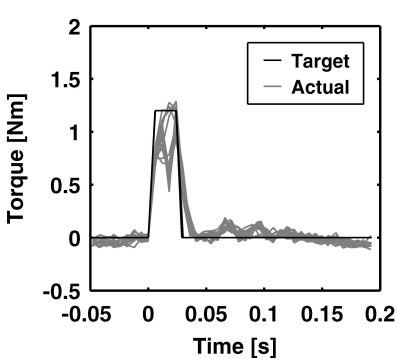

(a)

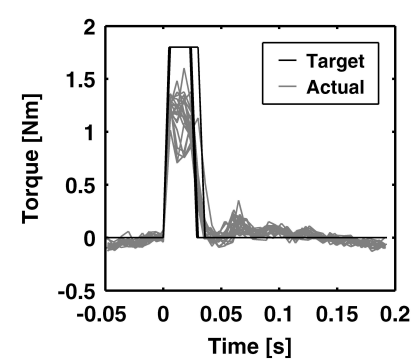

(b)
Fig. 15. Comparison of the target and actual torques for the actuation system for a pulsed torque waveform with frequency of $4.1 \mathrm{~Hz}$ and duty cycle of $10 \%$ for all hops in the test run with target positive torques of (a) $1.2 \mathrm{~N} \mathrm{~m}$ and (b) $1.8 \mathrm{Nm}$ with the negative pulse amplitude held at zero. The waveforms are synchronised on the rising edges of the target torque waveform.
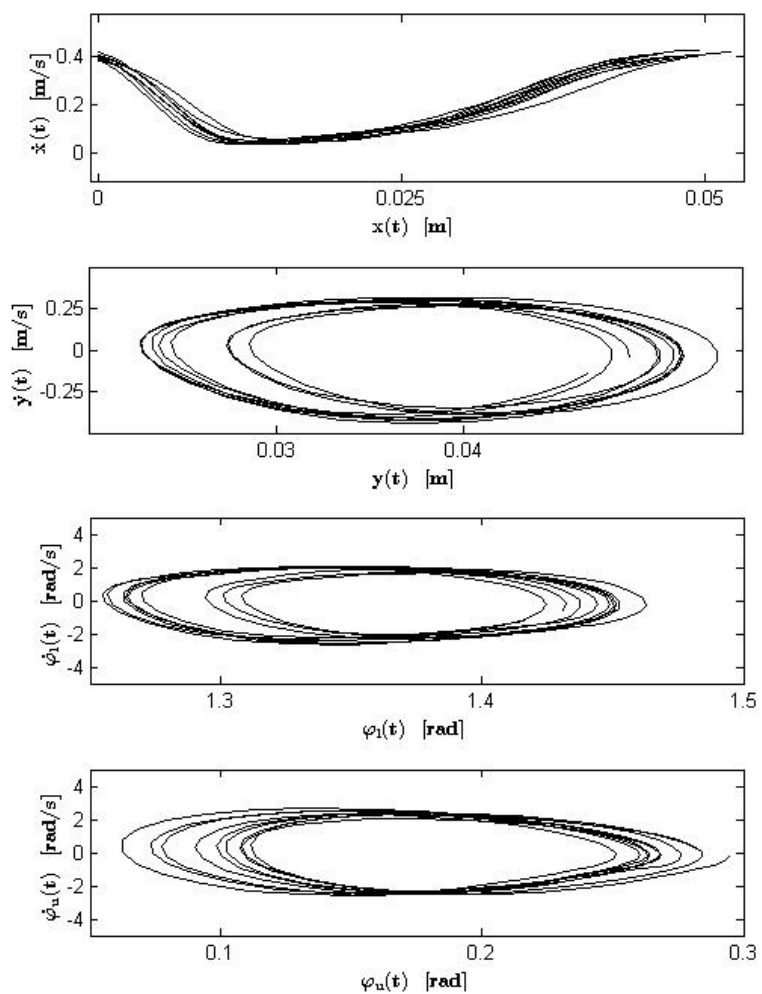

Fig. 16. Stability analysis of the pulsed torque case, with 10 consecutive stable hops. Both pulsed and sinusoidal cases showed comparable stability for successful hopping experiments, whereas the robot quickly collapsed for failed trials.

sinusoidal cases behave amid different forward velocities. The pulsed method allows the system to hop in lower speeds, as low as $0.03 \mathrm{~m} \mathrm{~s}^{-1}$, and as the hopping speed increases the CoT decreases, reaching 0.72. In our previous works [8], [9], [10], we have shown that a frequency within the range of $\pm 20 \%$ of the natural frequency is required to produce stable hopping, and the lack of lower sinusoidal speeds was expected. The total speed range from $0.12 \mathrm{~m} \mathrm{~s}^{-1}$ to $0.21 \mathrm{~m} \mathrm{~s}^{-1}$, where both 
cases had experimental results, shows that the pulsed method outperforms the sinusoidal method.

\section{DISCUSSION}

\section{A. Formulation of control input for locomotion}

The idea that cyclical control methods, such as sinusoidal [14], CPGs [23] or clocks [16], are more appropriate for cyclic locomotion has been around for more than 15 years, and an obvious reason is the widely used robotic actuation sources. The exploitation of natural dynamics [11] is only possible when elasticity exists, and the presence of cyclic patterns (sinusoid) creates an inefficient locomotion method when combined with such elasticity. In our work, we show that the walking patterns from sinusoidal (Fig. 13a) and pulsed (Fig. 13b) are similar, with minor differences concerning the start of the hopping, in spite of the control method and their efficiency being completely distinct, as demonstrated in Figs. 10 and 11. Our conclusions, rather than focusing on the adopted waveform, place in question the traditional choice of cyclical actuation methods over pulsed methods. Although our experiments adopted a square-waved pulse, we believe that different types of waves could have produced similar results, and the search for an optimal wave will be the aim of our future works.

Animals possess muscles as actuators, and electromyographic data shows that our muscle contractions are generated by series of spikes [15] and that the elasticity of the muscles filter these spikes to allow a smooth interaction with the environment. We hypothesise that the sinusoidal torque is an overcompensating measure and enforces control smoothness unnecessarily. In Figs. 10 and 11, a stark difference between both methods lies on the presence of negative work during the flight phase of the sinusoidal torque. While the pulsed solution passively explores the natural dynamic of the body to increase efficiency, in shrewd agreement with Remy [12], the sinusoidal method wastes energy while failing to exploit the morphology.

While in Fig. 5 we could see that each frequency was coupled to one specific amplitude in order to produce the lowest CoT, in Fig.7 the lowest CoT emerges from one single frequency over a broad range of amplitudes. A comparison between these experiments leads us to the same conclusion (Fig. 14), and we hypothesise that a pulsed torque exploits the natural elasticity offered by parallel elastic actuators.

Iida et al. [14] present a compass biped with open-loop sinusoidal control walking stably over obstacles, and hypothesise that phase locking ensures that the biped adapts to the uneven ground. Here, a similar phenomenon happens with the pulsed torque systematically taking place a few milliseconds before the takeoff in a completely open-loop system, as seen in Fig. 11. Although no explanation regarding the reason for occurrence of the phase locking was created, we ponder that this cyclic attractor state emerges from the coupling between mechanical and actuation dynamics.

In Remy [12] it is stated that speed and efficiency are directly related to the physical system, and we suppose that, in a similar fashion, morphology and control are directly related to produce stability. Phase locking is important for openloop controllers and our future work will concentrate on the emergence of this phenomenon.

\section{B. Pulsed torques show higher efficiency over different speeds}

In the seminal work of Srinivasan et al. [21] it is stated that impulsive torques are ideal for higher speeds. Analysing the variation of the CoT over different speeds (Fig. 14) we notice a better performance with pulsed torques than with sinusoidal ones, which partially agrees with [21]. Following the same trend observed within our simulations from the pulsed driving method (Fig. 7a), we infer that the results from [21] fortify our findings, and thus impulsive torques produce a higher energy efficiency for higher speeds.

The usage of experimental results to re-tune simulation parameters could have improved the accuracy of simulations for both pulsed and sinusoidal cases. Since our simulations and experiments adopted a broad search space, we believe that the exploratory aspect of the parameter search was significant enough to prove the superiority of pulsed cases over sinusoidal cases. A higher accuracy might be important to find the optimal control, and this will be one of the next tasks for our future works.

Beyond having a higher energy efficiency, the pulsed driving torque outperforms the sinusoidal driving torque by keeping the same efficiency and frequency over different velocities. In other words, a direct relationship between torque amplitude and output speed exists, simplifying the control for our hopping robot, which approximates to the phenomenon of "opening the throttle", observed in animals and coined by Shik et al. [22]. In Reis et al. [13] it is stated that the challenge is to "identify how larger locomotion velocities and behavioral diversity can be achieved while maintaining the energy efficiency", and our work successfully tackles the velocity aspect of this statement. Here we prove that a torque pulse can phase lock the system and couple amplitude with velocity when applied in agreement with the frequency and speed of the body.

\section{Damping, impact and friction}

When studying the energy efficiency of hoppers, the main concern is the energy loss between touchdown and take-off. As the body touches the floor the legs comply to convert the kinetic energy into elastic potential energy, and the pulsed driving method better exploited the "springy" behaviour of the system to reduce energy expenditure. The work from Seipel et al. [16] suggests that a higher forward velocity can be reached by increasing damping within the legs. Our results do not fortify this hypothesis, as the damping was not a controllable parameter. However, a brief analysis of Fig. 12 leads us to judiciously infer that a higher damping decreases the energy efficiency of the system, and the connection between energy and speed still remains to be established.

Remy [12] suggested that a non-optimal gait that is passively stable might actually be energetically superior than a highly optimised motion requiring heavy intervention. Our results agree with his claim, where the sinusoidal method was 
proved to be an overcompensating measure of the springy behaviour of the system. In this sense, less intervention (e.g. shutting down the sinusoidal torque for a preset time) would have produced a higher energy efficiency with potentially the same forward velocity.

Looking at the results from Fig. 6 and Fig. 8, we must note that it is impressive that the natural dynamics of the system and the control parameters are such that the brief pulses of torque occur at such times without the need for closedloop control. Such solutions utilise the sprung elements of the system merely as a periodic store of energy during the stance phase, rather than requiring true resonant behaviour. This pulsed method allows higher speeds with lower energy input, and consequently keeps a high efficiency while exploring the approximately flat relationship between CoT and speed for a wide range of locomotion speeds.

\section{CONCLUSions And Future Work}

In this work we compare a sinusoidal and a pulsed control method for a hopping robot. We use simulations from both cases to understand their differences, maximum speeds and energy expenditure. We test both control methods with a hopping robot and we find that the pulsed driving method outperforms the sinusoidal method in simulations and experiments between $0.12 \mathrm{~m} \mathrm{~s}^{-1}$ to $0.21 \mathrm{~m} \mathrm{~s}^{-1}$, as a comparison was not possible outside this range. Moreover, the pulsed driving method allowed the robot to continuously change its hopping speed while keeping a constant energy efficiency and hopping frequency. We prove that our gains in efficiency are due to lower losses during impact and damping, and that the sinusoidal controller produces negative work during flight phase, further reducing their efficiency.

These results suggest that there is an adequate time window within a locomotion gait for applying inputs to the system and achieve best locomotion efficiency. Sinusoidal inputs, such as those considered in previous works, add unnecessary energy to the system throughout the locomotion cycle and are detrimental to efficiency. Such findings will help shape the future of robotics by achieving higher energy efficiencies within legged robots while maintaining behaviour diversity.

\section{REFERENCES}

[1] V. A. Tucker, "The Energetic Cost of Moving About: Walking and running are extremely inefficient forms of locomotion. Much greater efficiency is achieved by birds, fish-and bicyclists." American Scientist, vol. 63 no. 4, pp. 413-419, July 1975.

[2] A. Kuo, "Choosing Your Steps Carefully", IEEE Robot. Automat. Mag vol. 14, pp. 18-29. 2007.

[3] A. Rosendo, X. Liu, M. Shimizu, and K. Hosoda, Stretch reflex improves rolling stability during hopping of a decerebrate biped system, Bioinspiration and Biomimetics, vol. 10 no. 1, pp. 016008, 2015.

[4] T. McGeer, Passive dynamic walking, International Journal of Robotic Research, vol. 9 no. 2, pp. 62-82, 1990.

[5] S. Seok, A. Wang, M.Y.M. Chuah, D.J. Hyun, J. Lee, D.M. Otten, J.H. Lang, and S. Kim Design principles for energy-efficient legged locomotion and implementation on the MIT Cheetah, IEEE Transaction on Mechatronics, vol. 20 no. 3, pp. 1117-1129, 2015.

[6] S. Rezazadeh, and J.W. Hurst, Toward step-by-step synthesis of stable gaits for underactuated compliant legged robots, 2015 IEEE International Conference on Robotics and Automation (ICRA), pp.4532-4538, 2015 .
[7] R. Blickhan, The spring-mass model for running and hopping, Journal of Biomechanics, vol. 22 no. 11-12, pp. 1217-1227, 2014.

[8] X. Yu and F. Iida, Minimalistic models of an energy-efficient verticalhopping robot, IEEE Transactions on Mechatronics, vol. 61 no. 2, pp. 1053-1062, 2014.

[9] F. Gunther, F. Giardina and F. Iida, Self-stable one-legged hopping using a curved foot, 2014 IEEE International Conference on Robotics and Automation (ICRA), pp.5133-5138, 2014.

[10] F. Gunther, Y. Shu and F. Iida, Parallel elastic actuation for efficient large payload locomotion, 2015 IEEE International Conference on Robotics and Automation (ICRA), pp.823-828, 2015.

[11] F. Iida and R. Pfeifer, Sensing through body dynamics, Robotics and Autonomous Systems, vol. 54 no. 8, pp. 631-640, 2006.

[12] C.D. Remy, Optimal exploitation of natural dynamics in legged locomotion, Ph.D. Thesis, ETHZ, 2011.

[13] M. Reis and F. Iida, An Energy-Efficient Hopping Robot Based on Free Vibration of a Curved Beam, IEEE Transaction on Mechatronics, vol. 19 no. 1, pp. 300-311, 2013

[14] F. Iida and R. Tedrake, Minimalistic control of biped walking in rought terrain, Autonomous Robots, vol. 28 no. 3, pp. 355-368, 2010.

[15] Y. P. Ivanenko, R. E. Poppele, and F. Lacquaniti, Five basic muscle activation patterns account for muscle activity during human locomotion, Journal of Physiology, vol. 556 no. 1, pp. 267-282, 2004.

[16] J. Seipel and P. Holmes, A simple model for clock-actuated legged locomotion, Regular and Chaotic Dynamics, vol. 12 no. 5, pp. 502-520, 2007.

[17] J. J. Moreau and P. D. Panagiotopoulos, Unilateral Contact and Dry Friction in Finite Freedom Dynamics, Nonsmooth Mechanics and Applications, CISM Courses and Lectures, Vol. 302, Springer Verlag, Wien $1988,1-82$.

[18] C. Glocker and C. Studer, Formulation and Preparation for Numerical Evaluation of Linear Complementarity Systems in Dynamics, Multibody System Dynamics, Vol. 13, no. 4, Springer Verlag, 2005, 447-463.

[19] J.-J. E. Slotine and W. Li, Applied Nonlinear Control. Englewood Cliffs, New Jersey: Prentice-Hall International, 1991.

[20] Newtons4th Ltd. (2012). Application Note - 014. 3 Phase 2 Wattmeter Power Measurements Explained [Online].

Available: www.newtons4th.com/wp-content/uploads/2014/04/APP0143-Phase-2-Wattmeter-Explained.pdf

[21] M. Srinivasan and A. Ruina, Computer optimization of minimal biped model discovers walking and running, Nature, vol. 439, pp. 72-75, 2006.

[22] M. Shik, L. Severin, and G.N. Orlovskii, Control of walking and running by means of electric stimulation of the midbrain, Biofyzika, vol. 11, pp. 659,1966

[23] Y. Fukuoka, H. Kimura, Y. Hada and K. Takase, Adaptive dynamic walking of a quadruped robot 'Tekken' on irregular terrain using a neural system model, 2003 IEEE International Conference on Robotics and Automation (ICRA), pp.2037-2042, 2003. 